\title{
Reducing the exome search space for Mendelian diseases using genetic linkage analysis of exome genotypes
}

\author{
Katherine R Smith ${ }^{1 *}$, Catherine J Bromhead ${ }^{1}$, Michael S Hildebrand ${ }^{2}$, A Eliot Shearer ${ }^{2,3}$, Paul J Lockhart ${ }^{4,5}$, \\ Hossein Najmabadi ${ }^{6}$, Richard J Leventer ${ }^{4,7,8}$, George McGillivray ${ }^{4}$, David J Amor ${ }^{4,7}$, Richard J Smith ${ }^{2,3,9}$ and \\ Melanie Bahlo ${ }^{1,10}$
}

\begin{abstract}
Many exome sequencing studies of Mendelian disorders fail to optimally exploit family information. Classical genetic linkage analysis is an effective method for eliminating a large fraction of the candidate causal variants discovered, even in small families that lack a unique linkage peak. We demonstrate that accurate genetic linkage mapping can be performed using SNP genotypes extracted from exome data, removing the need for separate array-based genotyping. We provide software to facilitate such analyses.
\end{abstract}

\section{Background}

Whole exome sequencing (WES) has recently become a popular strategy for discovering potential causal variants in individuals with inherited Mendelian disorders, providing a cost- effective, fast-track approach to variant discovery. However, a typical human genome differs from the reference genome at over 10,000 potentially functional sites [1]; identifying the disease-causing mutation among this plethora of variants can be a significant challenge. For this reason, exome sequencing is often preceded by genetic linkage analysis, which allows variants outside of linkage peaks to be excluded. The linkage peaks delineate tracts of identity by descent sharing that match the proposed genetic model. This combination strategy has been successfully used to identify variants causing autosomal dominant [2-4] and recessive [5-11] diseases, as well as those affecting quantitative traits [12-14]. Linkage analysis has also been used in conjunction with whole genome sequencing (WGS) [15].

Other WES studies have not performed formal linkage analysis, but have nonetheless considered inheritance information, such as searching for large regions of homozygosity shared by affected family members using

\footnotetext{
* Correspondence: katsmith@wehi.edu.au

'Bioinformatics Division, The Walter and Eliza Hall Institute of Medical

Research, 1G Royal Parade, Parkville, Victoria 3052, Australia Full list of author information is available at the end of the article
}

genotypes obtained from genotyping arrays [16-18] or exome data $[19,20]$. This method does not incorporate genetic map or allele frequency information, which could help to eliminate regions from consideration, and is applicable only to recessive diseases resulting from consanguinity. Recently, it has been suggested that identity by descent regions be identified from exome data using a non-homogeneous hidden Markov model (HMM), allowing variants outside these regions to be eliminated $[21,22]$. This method incorporates genetic map information but not allele frequency information and requires a strict genetic model (recessive and fully penetrant) and sampling scheme (exomes of two or more affected siblings must be sequenced). It would be suboptimal for use with diseases resulting from consanguinity, for which filtering by homozygosity by descent would be more effective than filtering by identity by descent. Finally, several WES studies have been published that make no use of inheritance information whatsoever, despite the fact that DNA from other informative family members was available [23-31].

Classical linkage analysis using the multipoint LanderGreen algorithm [32], which is a HMM, incorporates genetic map and allele frequency information and allows for great flexibility in the disease model. Unlike the methods just mentioned, linkage analysis allows dominant, recessive or X-linked inheritance models, as well
Ciomed Central 
as permitting variable penetrances, non-parametric analysis and formal haplotype inference. There are few constraints upon the sampling design, with unaffected individuals able to contribute information to parametric linkage analyses. The Lander-Green algorithm has produced many important linkage results, which have facilitated the identification of the underlying disease-causing mutations.

We investigated whether linkage analysis using the Lander-Green algorithm could be performed using genotypes inferred from WES data, removing the need for the array-based genotyping step [33]. We inferred genotypes at the location of HapMap Phase II SNPs, [34] as this resource provides comprehensive annotation, including the population allele frequencies and genetic map positions required for linkage analysis. We adapted our existing software [35] to extract HapMap Phase II SNP genotypes from WES data and format them for linkage analysis.

We anticipated two potential disadvantages to this approach. Firstly, exome capture only targets exonic SNPs, resulting in gaps in marker coverage outside of exons. Secondly, genotypes obtained using massively parallel sequencing (MPS) technologies such as WES tend to have a higher error rate than those obtained from genotyping arrays [36]. The use of erroneous genotypes in linkage analyses may reduce power to detect linkage peaks or result in false positive linkage peaks [37].

We compared the results of linkage analysis using array-based and exome genotypes for three families with different neurological disorders showing Mendelian inheritance (Figure 1). We sequenced the exomes of two affected siblings from family $\mathrm{M}$, an Anglo-Saxon ancestry family showing autosomal dominant inheritance.
The exome of a single affected individual, the offspring of first cousins, from Iranian family A was sequenced, as was the exome of a single affected individual, the offspring of parents thought to be first cousins once removed, from the Pakistani family $\mathrm{T}$. Families A and $\mathrm{T}$ showed recessive inheritance. Due to the consanguinity present in these families, we can perform linkage analysis using genotypes from a single affected individual, a method known as homozygosity mapping [33].

\section{Results and discussion}

\section{Exome sequencing coverage of HapMap Phase II SNPs}

Allele frequencies and genetic map positions were available for 3,269,163 HapMap Phase II SNPs that could be translated to UCSC hg19 physical coordinates. The Illumina TruSeq platform used for exome capture targeted 61,647 of these SNPs (1.89\%). After discarding indels and SNPs whose alleles did not match the HapMap annotations, a median 56,931 (92.3\%) of targeted SNPs were covered by at least five high-quality reads (Table 1). A median of 64,065 untargeted HapMap Phase II SNPs were covered by at least five reads; a median $78 \%$ of these untargeted SNPs were found to lie within 200 bp of a targeted feature, comprising a median $57 \%$ of all untargeted HapMap SNPs within 200 bp of a targeted feature.

In total, we obtained a minimum of 117,158 and a maximum of 133,072 SNP genotypes from the four exomes. The array-based genotyping interrogated 598,821 genotypes for A-7 and T-1 (Illumina Infinium HumanHap610W-Quad BeadChip) and 731,306 genotypes for M-3 and M-4 (Illumina OmniExpress BeadChip). Table 2 compares the inter-marker distances between exome genotypes for each sample to those for the genotyping array. The exome genotypes have much
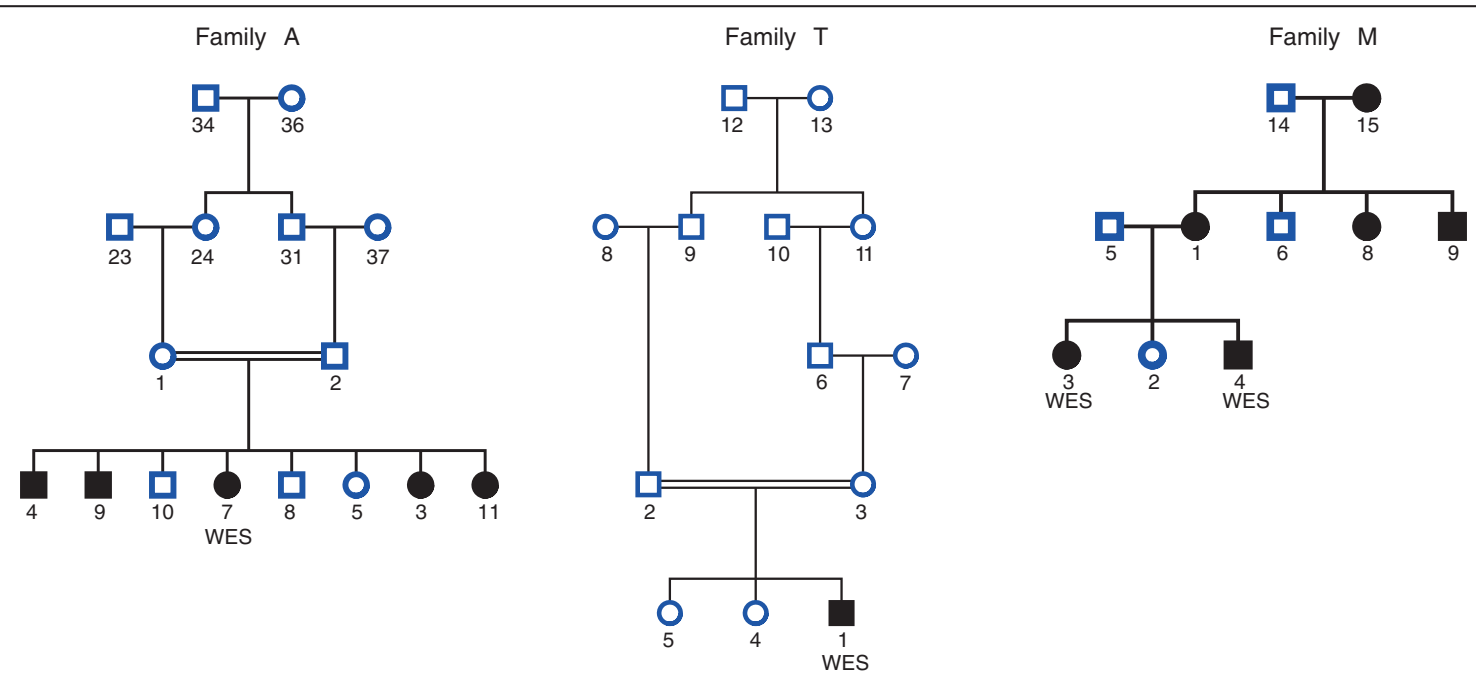

Figure 1 Partial pedigrees for families $A, T$ and $M$ 
Table 1 Number of HapMap Phase II SNPs covered $\geq 5$ by distance to targeted base

\begin{tabular}{lccccc}
\hline Distance to & \multicolumn{2}{c}{ Number of SNPs (\%) } & \multicolumn{2}{c}{ HapMap } \\
targeted base & M-3 & M-4 & A-7 & T-1 & Phase II (N) \\
\hline y bp & $56,648(91.9)$ & $56,835(92.2)$ & $57,027(92.5)$ & $58,142(94.3)$ & 61,647 \\
1 to $200 \mathrm{bp}$ & $50,077(56.7)$ & $50,805(57.5)$ & $46,144(52.2)$ & $57,923(65.6)$ & 88,349 \\
$>$ 200 bp & $13,683(0.4)$ & $13,565(0.4)$ & $13,987(0.4)$ & $17,007(0.5)$ & $3,119,167$ \\
Total & $120,408(3.7)$ & $121,205(3.7)$ & $117,158(3.6)$ & $133,072(4.1)$ & $3,269,163$ \\
\hline
\end{tabular}

The denominator for percentages is the total number of HapMap Phase II SNPs in that distance category.

more variable inter-marker distances than the genotyping arrays, with a smaller median value.

\section{Optimization of genotype concordance}

We inferred genotypes at the positions of SNPs located on the genotyping array used for each individual so that we could investigate genotype concordance between the two technologies. We found that ambiguous (A/T or $\mathrm{C} /$ G SNPs) comprised a high proportion of SNPs with discordant genotypes, despite being a small proportion of SNPs overall. For example, for A-7 at coverage $\geq 5$ and $t=0.5$ (see below), 77\% (346 of 450) of discordant SNPs were ambiguous SNPs, while ambiguous SNPs composed just $2.7 \%$ of all SNPs (820 of 30,279). Such SNPs are prone to strand annotation errors, as the two alleles are the same on both strands of the SNP. We therefore discarded ambiguous SNPs, which left 29,459 to 52,892 SNPs available for comparison (Table 3).

Several popular genotype-calling algorithms for MPS data require the prior probability of a heterozygous genotype to be specified $[38,39]$. We investigated the effect of varying this parameter, $t$, upon concordance of genotyping array and WES genotypes (given WES coverage $\geq$ 5; Table 3). Increasing this value from the default 0.001 results in a modest improvement in the percentage of WES genotypes being correctly classified, with most of the improvement occurring between $t=0.001$ and $t=$ 0.05 . The highest concordance is achieved at $t=0.5$, where all four samples achieve $99.7 \%$ concordance, compared to 98.7 to $98.9 \%$ concordance at the default $t=$ 0.001 .

Table 2 Intermarker distances for the two genotyping arrays and for exome genotypes covered $\geq 5$

\begin{tabular}{lrrr}
\hline & Median & 1st quartile & 3rd quartile \\
\hline Illumina OmniExpress & 2,233 & 814 & 5,125 \\
Illumina 610 & 2,744 & 1,019 & 6,027 \\
M-3 & 1,853 & 236 & 11,390 \\
M-4 & 1,830 & 235 & 11,260 \\
A-7 & 1,943 & 240 & 12,000 \\
T-1 & 1,647 & 227 & 10,210 \\
\hline
\end{tabular}

Intermarker distances are in base pairs.
We note that $t=0.5$ may not be optimal for calling SNP genotypes on haploid chromosomes. At $t=0.5$, the male M-4 had five $\times$ chromosome genotypes erroneously called as heterozygous out of $1,026(0.49 \%)$, while the male T-1 had one such call out of 635 genotypes $(0.16 \%)$. The same SNPs were not called as heterozygous by the genotyping arrays. No heterozygous $\times$ chromosome calls were observed at the default value of $t=0.001$.

\section{Linkage analysis and LOD score concordance}

Prior to performing linkage analysis on exome and array SNP genotypes, we selected one SNP per $0.3 \mathrm{cM}$ to ensure linkage equilibrium while retaining a set of SNPs dense enough to effectively infer inheritance. The resulting subsets of WES genotypes (Table 4) contained 8,016 to 8,402 SNPs with average heterozygosities of 0.40 or 0.41 among the CEPH HapMap genotypes, obtained from Utah residents with ancestry from northern and western Europe (CEU). The resulting subsets of array genotypes (Table 4) contained more SNPs (12,173 to 12,243), with higher average heterozygosities ( 0.48 or 0.49 ).

Despite this difference, there was good agreement between LOD scores achieved at linkage peaks using the different sets of genotypes (Figure 2, Table 5). The median difference between the WES and array LOD scores across positions where either achieved the maximum score was close to zero for all three families (range -0.0003 to -0.002$)$. The differences had a $95 \%$ empirical interval of $(-0.572,0.092)$ for family $A$, with the other two families achieving narrower intervals (Table 5).

\section{Efficacy of filtering identified variants by location of linkage peaks}

If our genetic model is correct, then variants lying outside of linkage peaks cannot be the causal mutation and can be discarded, thus reducing the number of candidate disease-causing variants. Table 6 lists the number of nonsynonymous exonic variants (single nucleotide variants or indels) identified in each exome, as well as the number lying with linkage peaks identified using WES genotypes. The percentage of variants eliminated depends upon the power of the pedigree being studied: $81.2 \%$ of variants are eliminated for the dominant family $\mathrm{M}$, which is not very powerful; $94.5 \%$ of variants are 
Table 3 Increasing the prior heterozygous probability modestly improves concordance between exome and array genotypes

\begin{tabular}{lcccc}
\hline $\boldsymbol{t}$ & $\mathbf{M - 3} \mathbf{( N = 5 2 , 6 1 7 )}$ & $\mathbf{M - 4} \mathbf{( N = \mathbf { 5 2 , 8 9 2 } )}$ & $\mathbf{A - 7}(\mathbf{N}=\mathbf{2 9 , 4 5 9 )}$ & $\mathbf{T}-\mathbf{1}(\mathbf{N}=\mathbf{3 2 , 7 6 3 )}$ \\
\hline 0.00001 & 0.9737 & 0.9734 & 0.9698 & 0.9741 \\
0.001 (default) & 0.9882 & 0.9874 & 0.9865 & 0.9885 \\
0.01 & 0.9927 & 0.9926 & 0.9918 & 0.9925 \\
0.05 & 0.9951 & 0.9950 & 0.9942 & 0.9945 \\
0.1 & 0.9958 & 0.9958 & 0.9950 & 0.9952 \\
0.2 & 0.9968 & 0.9965 & 0.9958 & 0.9961 \\
0.3 & 0.9971 & 0.9968 & 0.9961 & 0.9964 \\
0.4 & 0.9973 & 0.9971 & 0.9964 & 0.9968 \\
0.5 & 0.9974 & 0.9973 & 0.9965 & 0.9969 \\
\hline
\end{tabular}

Proportion of SNPs where WES and genotyping array genotypes are concordant for the four exomes, for varying values of $t$ (prior probability of a heterozygous genotype). Conditional on coverage with $\geq 5$ reads.

eliminated for the recessive, consanguineous family A; while $99.43 \%$ of variants are eliminated for the more distantly consanguineous, recessive family $\mathrm{T}$. Hence, linkage analysis substantially reduces the fraction of variants identified that are candidates for the disease-causing variant of interest.

\section{Conclusions}

Linkage analysis is of great potential benefit to WES studies that aim to discover genetic variants resulting in Mendelian disorders. As variants outside of linkage peaks can be eliminated, it reduces the number of identified variants that need to be investigated further. Linkage analysis of WES genotypes provides information regarding the location of the disease locus to be extracted from WES data even if the causal variant is not captured, suggesting regions of interest that may be targeted in follow-up studies. However, many such studies are being published that employ less sophisticated substitutes for linkage analysis or do not consider inheritance information at all. Anecdotal evidence suggests that a substantial proportion of MPS studies of individuals with Mendelian disorders fail to identify a causal variant, though an exact number is not known due to publication bias.

We describe how to extract HapMap Phase II SNP genotypes from massively parallel sequencing data,

Table 4 Number and average heterozygosity of array and WES SNPs selected for linkage analysis

\begin{tabular}{lcccccc}
\hline & \multicolumn{2}{c}{ M-3 and M-4 } & \multicolumn{2}{c}{ A-7 } & \multicolumn{2}{c}{ T-1 } \\
\cline { 2 - 7 } & WES & Array & WES & Array & WES & Array \\
\hline SNPs available & 114,681 & 677,144 & 117,158 & 593,638 & 133,071 & 587,680 \\
SNPs selected & 8,016 & 12,173 & 8,135 & 12,243 & 8,402 & 12,194 \\
Average & 0.40 & 0.49 & 0.40 & 0.48 & 0.41 & 0.48 \\
heterozygosity & & & & & & \\
\hline Averge
\end{tabular}

Average heterozygosity refers to the HapMap CEU population and not to the individual being studied. For $\mathrm{M}-3$ and $\mathrm{M}-4$, 'SNPs available' is the number of SNPs covered $\geq 5$ in both individuals. providing software to facilitate this process and generate files ready to be analyzed by popular linkage programs. Our method allows linkage analysis to be performed without requiring genotyping arrays. The flexibility of linkage analysis means that our method can be applied to any disease model and a variety of sampling schemes, unlike existing methods of considering inheritance information for WES data. Linkage analysis incorporates population allele frequencies and genetic map positions, which allows superior identification of statistically unusual sharing of haplotypes between affected individuals in a family.

We demonstrate linkage using WES genotypes for three small nuclear families - a dominant family from which two exomes were sequenced and two consanguineous families from which a single exome was sequenced. As these families are not very powerful for linkage analysis, multiple linkage peaks with relatively low LOD scores were identified. Nonetheless, discarding variants outside of the linkage peaks eliminated between $81.2 \%$ and $99.43 \%$ of all nonsynonymous exonic variants detected in these families. The number of variants remaining could be reduced further by applying standard strategies, such as discarding known SNPs with minor allele frequencies above a certain threshold. Our work demonstrates the value of considering inheritance information, even in very small families that may consist, at the extreme, of a single inbred individual. As the price of exome sequencing falls, it will become feasible to sequence more individuals from each family, resulting in fewer linkage peaks with higher LOD scores.

Exome capture using current technologies yields large numbers of useful SNPs for linkage mapping. Over half of all SNPs covered by five or more reads were not targeted by the exome capture platform. Approximately $78 \%$ of these captured untargeted SNPs lay within $200 \mathrm{bp}$ of a targeted feature. This reflects the fact that fragment lengths typically exceed probe lengths, resulting in flanking sequences at both ends of 
A
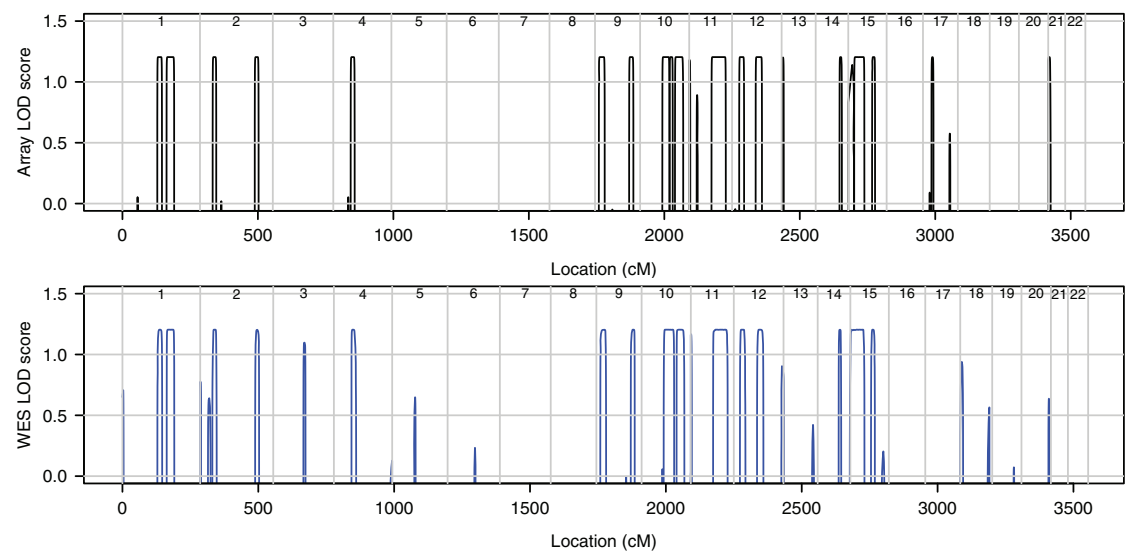

$\mathrm{T}$
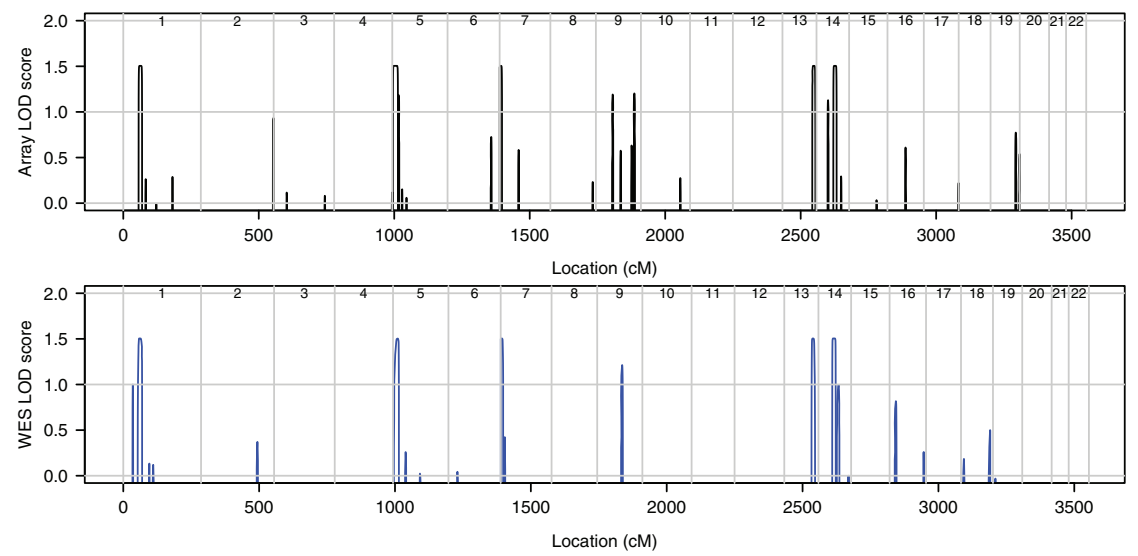

M
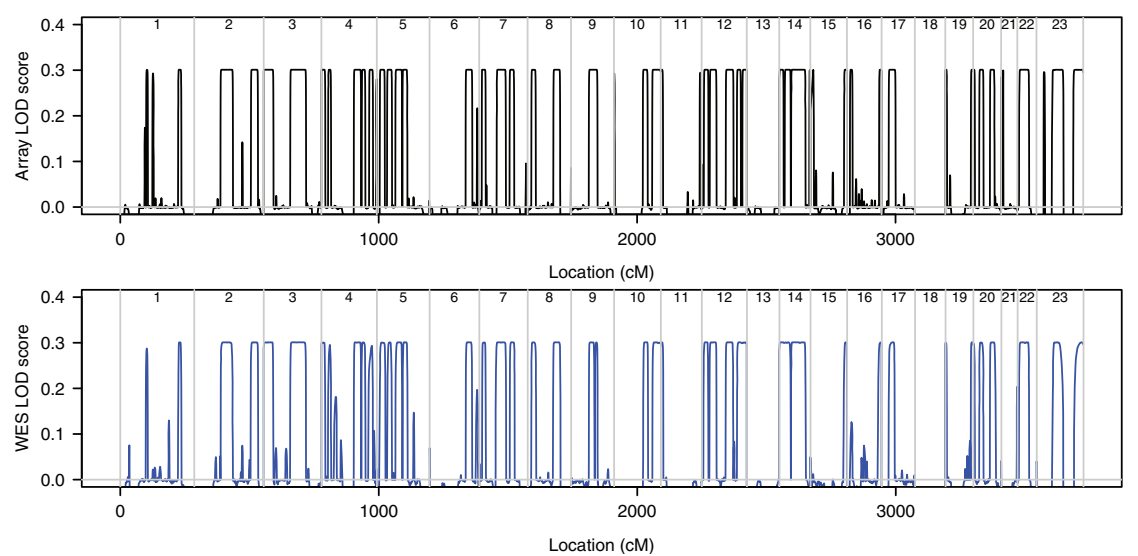

Figure 2 Genome-wide comparison of LOD scores using array-based and WES-derived genotypes for families $A, T$ and $M$.

a probe or bait being captured and sequenced. The serendipitous result is that a substantial number of non-exonic SNPs become available, which can and should be used for linkage analysis.

We found that setting the prior probability of heterozygosity to 0.5 during genotype inference resulted in the best concordance between WES and array genotypes.
The authors of the MAQ SNP model recommend using $t=0.2$ for inferring genotypes at known SNPs [38], while the default value used to detect variants is $t=$ 0.001 . Our results highlight the need to tailor this parameter to the specific application, either genotyping or rare variant detection. Although we anticipated WES genotypes being less accurate than array genotypes, all 
Table 5 Distribution of LOD score differences (WES array) at linkage peaks

\begin{tabular}{lccc}
\hline Family & Median & 2.5th centile & 97.5th centile \\
\hline A & -0.0005 & -0.572 & 0.092 \\
$T$ & -0.002 & -0.390 & 0.035 \\
$M$ & -0.0003 & -0.117 & 0.0034 \\
\hline
\end{tabular}

Summary of differences at analysis positions where either the WES or the array LOD scores reach their genome-wide maximum.

four samples achieved a high concordance of $99.7 \%$ for SNPs covered by five or more reads at $t=0.5$

We found that LOD scores obtained from WES genotypes agreed well with those obtained from array genotypes from the same individual(s) at the location of linkage peaks, with the median difference in LOD score zero to two or three decimal places for all three families. This was despite the fact that the array-based genotype sets used for analysis contained more markers and had higher average heterozygosities than the corresponding WES genotype sets, reflecting the fact that genotyping arrays are designed to interrogate SNPs with relatively high minor allele frequencies that are relatively evenly spaced throughout the genome. By contrast, genotypes extracted from WES data tend to be clustered around exons, resulting in fewer and less heterozygous markers after pruning to achieve linkage equilibrium. We conclude that if available, array-based genotypes from a high resolution SNP array are preferable to WES genotypes; but if not, linkage analysis of WES genotypes produces acceptable results.

Once WGS is more economical, we will be able to perform linkage analysis using genotypes extracted from WGS data, which will obviate the problem of gaps in SNP coverage outside of exons. The software tools we provide can accommodate WGS genotypes without requiring modification. In the future, initiatives such as the 1000 Genomes Project [1] may provide populationspecific allele frequencies for SNPs not currently included in HapMap, further increasing the number of SNPs available for analyses, as well as the number of populations studied.

The classic Lander-Green algorithm requires markers to be in linkage equilibrium [40]. Modeling linkage disequilibrium would allow incorporation of all markers without the need to select a subset of markers in linkage equilibrium. This would allow linkage mapping using distant relationships, such as distantly inbred individuals who would share a sub-linkage $(<1 \mathrm{cM})$ tract of DNA homozygous by descent. Methods that incorporate linkage disequilibrium have already been proposed, including a variable length HMM that can be applied to detect distantly related individuals [41]. Further work is being targeted towards approximations of distant relationships to connect sets of related pedigrees [42]. These methods will extract the maximum information from MPS data from individuals with inherited diseases.

We have integrated the relatively new field of MPS in families with classical linkage analysis. Where feasible, we strongly advocate the use of linkage mapping in combination with MPS studies that aim to discover variants causing Mendelian disorders. This approach does not require purpose-built HMMs, but can utilize existing software implementations of the Lander-Green algorithm. Where genotyping array genotypes are not available, we recommend utilizing MPS data to their full capacity by using MPS genotypes to perform linkage analysis. This will reduce the number of candidate disease-causing variants that need to be evaluated further. Should the causal variant not be identified by a WES study, linkage analysis will highlight regions of the genome where targeted resequencing is most likely to identify this variant.

\section{Materials and methods}

Informed consent, DNA extraction and array-based genotyping

Written informed consent was provided by the four participants or their parents. Ethics approval was provided by the Royal Children's Hospital Research Ethics Committee (HREC reference number 28097) in Melbourne. Genomic DNA was extracted from participants' blood samples using the Nucleon ${ }^{\mathrm{TM}}$ BACC Genomic DNA Extraction Kit (GE Healthcare, Little Chalfont, Buckinghamshire, England).

All four individuals were genotyped using Illumina Infinium HumanHap610W-Quad BeadChip (A-7, T-1) or OmniExpress (M-3, M-4) genotyping arrays (fee for service, Australian Genome Research Facility, Melbourne, Victoria, Australia). These arrays interrogate

Table 6 Efficacy of variant elimination due to linkage peak filtering

\begin{tabular}{|c|c|c|c|c|c|c|}
\hline Family & Model & Consanguinity & $\begin{array}{l}\text { Number of } \\
\text { linkage peaks }\end{array}$ & $\begin{array}{l}\text { Max } \\
\text { LOD }\end{array}$ & $\begin{array}{c}\text { Number of not } \\
\text { synonymous exonic } \\
\text { variants }\end{array}$ & $\begin{array}{c}\text { Number of (\%) not synonymous exonic } \\
\text { variants in linkage regions }\end{array}$ \\
\hline A & Recessive & First cousin offspring & 15 & 1.2 & 10,982 & $604(5.50)$ \\
\hline T & Recessive & $\begin{array}{l}\text { First cousins once } \\
\text { removed offspring }\end{array}$ & 5 & 1.51 & 11,353 & $65(0.57)$ \\
\hline M & Dominant & None & 41 & 0.3 & 13,186 & 2,478 (18.79) \\
\hline
\end{tabular}


598,821 and 731,306 SNPs respectively, with 342,956 markers in common. Genotype calls were generated using version 6.3 .0 of the GenCall algorithm implemented in Illumina BeadStudio. A GenCall score cutoff (nocall threshold) of 0.15 was used.

\section{Exome capture, sequencing and alignment}

Target DNA for the four individuals was captured using Illumina TruSeq, which is designed to capture a target region of $62,085,286$ bp (2.00\% of the genome), and sequenced using an Illumina HiSeq machine (fee for service, Axeq Technologies, Rockville, MD, United States). Individual T-1 was sequenced using one-quarter of a flow cell lane while the other three individuals were sequenced using one-eighth of a lane. Paired-end reads of $110 \mathrm{bp}$ were generated.

Reads were aligned to UCSC hg19 using Novoalign version 2.07.05 [43]. Quality score recalibration was performed during alignment, and reads that aligned to multiple locations were discarded. Following alignment, presumed PCR duplicates were removed using MarkDuplicates.jar from Picard [44]. Table S1 in Additional file 1 shows the number of reads at each stage of processing, while Tables S2 and S3 in the same file show coverage statistics for the four exomes.

\section{WES genotype inference and linkage analysis}

SNP genotypes were inferred from WES data using the samtools mpileup and bcftools view commands from release 916 of the SAMtools package [45], which infers genotypes using a revised version of the MAQ SNP model [38]. We required base quality and mapping quality $\geq 13$. SAMtools produces a variant call format (VCF) file, from which we extracted genotypes using a Perl script.

These genotypes were formatted for linkage analysis using a modified version of the Perl script linkdatagen.pl [35] with an annotation file prepared for HapMap Phase II SNPs. This script chose one SNP per $0.3 \mathrm{cM}$ to be used for analysis, with SNPs selected to maximize heterozygosity according to CEU HapMap genotypes [34]. Array-based genotypes were prepared for linkage analysis in the same way, using annotation files for the appropriate array.

The two Perl scripts used to extract genotypes from VCF files and format them for linkage analysis are freely available on our website [46], as is the annotation file for HapMap Phase II SNPs. Users may also download VCF files containing WES SNP genotypes for the four individuals described here (both for HapMap Phase II and genotyping array SNPs), as well as files containing genotyping array genotypes for comparison.

Multipoint parametric linkage analysis using WES and array genotypes was performed using MERLIN [47]. A population disease allele frequency of 0.00001 was specified, along with a fully penetrant recessive (family A, family $\mathrm{T}$ ) or dominant (family $\mathrm{M}$ ) genetic model. LOD scores were estimated at positions spaced $0.3 \mathrm{cM}$ apart, and CEU allele frequencies were used.

\section{WES variant detection}

SAMtools mpileup/bcftools was also used to detect variants from the reference sequence with the default setting of $t=0.001$. Variants were annotated by ANNOVAR [48] using the UCSC Known Gene annotation. For the purposes of filtering variants, linkage peaks were defined as the intervals in which the genome-wide maximum LOD score was obtained, plus $0.3 \mathrm{cM}$ on either side.

\section{Additional material}

Additional file 1: Supplementary tables.

\section{Abbreviations}

bp: base pair; HMM: hidden Markov model; MPS: massively parallel sequencing; SNP: single nucleotide polymorphism; VCF: variant call format; WES: whole exome sequencing; WGS: whole genome sequencing.

\section{Acknowledgements}

We acknowledge Kate Pope, Hayley Mountford and Elizabeth Fitzpatrick (Accelerated Gene Identification Project, Murdoch Childrens Research Institute) for assistance with families A, T and M. This work was supported by an Australian Research Council (ARC) Future Fellowship (MB), an NHMRC Program Grant (MB, DJA), NIH-NIDCD grant RO1 DCOO2842 (RJHS), NHMRC overseas biomedical postdoctoral training fellowship 546943 (MSH), a Doris Duke Fellowship (AES) and the Victorian Government's Operational Infrastructure Support Program (PL, RJL, GM, DJA). Funding sources had no role any of the following: design of the study; the collection, analysis, and interpretation of data; the writing of the manuscript; and the decision to submit the manuscript for publication.

\section{Author details}

${ }^{1}$ Bioinformatics Division, The Walter and Eliza Hall Institute of Medical Research, $1 \mathrm{G}$ Royal Parade, Parkville, Victoria 3052, Australia. ${ }^{2}$ Department of Otolaryngology-Head and Neck Surgery, University of lowa, lowa City, lowa 52242, USA. ${ }^{3}$ Department of Molecular Physiology and Biophysics, University of lowa Carver College of Medicine, lowa City, IA 52242, USA. ${ }^{4}$ Murdoch Childrens Research Institute, Royal Children's Hospital, Parkville, Victoria 3052, Australia. ${ }^{5}$ Bruce Lefroy Centre for Genetic Health Research, Murdoch Childrens Research Institute, Royal Children's Hospital, Parkville, Victoria 3052, Australia. ${ }^{6}$ Genetics Research Center, University of Social Welfare and Rehabilitation Sciences, Tehran 19834, Iran. ${ }^{7}$ Department of Paediatrics, University of Melbourne, Royal Children's Hospital, Parkville, Victoria 3052, Australia. ${ }^{8}$ Children's Neuroscience Centre, Royal Children's Hospital, Parkville, Victoria 3052, Australia. Interdepartmental PhD Program in Genetics, University of lowa, lowa City, lowa 52242, USA. ${ }^{10}$ Department of Mathematics and Statistics, The University of Melbourne, Parkville, Victoria 3010, Australia

\section{Authors' contributions}

KRS conceived of the study and performed all analyses described in the article. MB provided guidance and ideas. CJB wrote software tools. MSH, AES, and RJHS performed whole exome sequencing. MSH performed arraybased SNP genotyping. RJHS, RJL, HN, GM and DJA collected families and clinical data. PJL contributed reagents and materials. KRS and MB drafted the initial article. All authors discussed the results and commented on the manuscript. 
Received: 8 April 2011 Revised: 28 July 2011

Accepted: 13 September 2011 Published: 14 September 2011

\section{References}

1. Durbin RM, Abecasis GR, Altshuler DL, Auton A, Brooks LD, Gibbs RA, Hurles ME, McVean GA, 1000 Genomes Project Consortium: A map of human genome variation from population-scale sequencing. Nature 2010, 467:1061-1073.

2. Johnson JO, Mandrioli J, Benatar M, Abramzon Y, Van Deerlin VM, Trojanowski JQ, Gibbs JR, Brunetti M, Gronka S, Wuu J, Ding J, McCluskey L, Martinez-Lage M, Falcone D, Hernandez DG, Arepalli S, Chong S, Schymick JC, Rothstein J, Landi F, Wang Y-D, Calvo A, Mora G, Sabatelli M, Monsurrò , Rosaria Maria, Battistini S, Salvi F, Spataro R, Sola P, Borghero G, et al: Exome Sequencing Reveals VCP Mutations as a Cause of Familial ALS. Neuron 2010, 68:857-864.

3. Wang $J$, Yang $X$, Xia $K$, Hu ZM, Weng $L$, Jin $X$, Jiang $H$, Zhang $P$, Shen $L$, Feng Guo J, Li N, Li YR, Lei LF, Zhou J, Du J, Zhou YF, Pan Q, Wang J, Wang J, Li RQ, Tang BS: TGM6 identified as a novel causative gene of spinocerebellar ataxias using exome sequencing. Brain 2010, 133:3510-3518

4. Southgate L, Machado RD, Snape KM, Primeau M, Dafou D, Ruddy DM, Branney PA, Fisher M, Lee GJ, Simpson MA, He Y, Bradshaw TY, Blaumeiser B, Winship WS, Reardon W, Maher ER, FitzPatrick DR, Wuyts W, Zenker M, Lamarche-Vane N, Trembath RC: Gain-of-Function Mutations of ARHGAP31, a Cdc42/Rac1 GTPase Regulator, Cause Syndromic Cutis Aplasia and Limb Anomalies. The American Journal of Human Genetics 2011, 88:574-585.

5. Bilguvar K, Ozturk AK, Louvi A, Kwan KY, Choi M, Tatli B, Yalnizoglu D, Tuysuz B, Caglayan AO, Gokben S, Kaymakcalan H, Barak T, Bakircioglu M, Yasuno K, Ho W, Sanders S, Zhu Y, Yilmaz S, Dincer A, Johnson MH, Bronen RA, Kocer N, Per H, Mane S, Pamir MN, Yalcinkaya C, Kumandas S, Topcu M, Ozmen M, Sestan N, et al: Whole-exome sequencing identifies recessive WDR62 mutations in severe brain malformations. Nature 2010 467:207-210

6. Bolze A, Byun M, McDonald D, Morgan NV, Abhyankar A, Premkumar L, Puel A, Bacon CM, Rieux-Laucat F, Pang K, Britland A, Abel L, Cant A, Maher ER, Riedl SJ, Hambleton S, Casanova J-L: Whole-Exome-SequencingBased Discovery of Human FADD Deficiency. Am J Hum Genet 2010, 87:873-881.

7. Kalay E, Yigit G, Aslan Y, Brown KE, Pohl E, Bicknell LS, Kayserili H, Li Y, Tuysuz B, Nurnberg G, Kiess W, Koegl M, Baessmann I, Buruk K, Toraman B, Kayipmaz S, Kul S, Ikbal M, Turner DJ, Taylor MS, Aerts J, Scott C, Milstein K, Dollfus H, Wieczorek D, Brunner HG, Hurles M, Jackson AP, Rauch A, Nurnberg $P$, et al: CEP152 is a genome maintenance protein disrupted in Seckel syndrome. Nat Genet 2011, 43:23-26.

8. Otto EA, Hurd TW, Airik R, Chaki M, Zhou W, Stoetzel C, Patil SB, Levy S, Ghosh AK, Murga-Zamalloa CA, van Reeuwijk J, Letteboer SJF, Sang L, Giles RH, Liu Q, Coene KLM, Estrada-Cuzcano A, Collin RWJ, McLaughlin HM, Held S, Kasanuki JM, Ramaswami G, Conte J, Lopez I, Washburn J, Macdonald J, Hu J, Yamashita Y, Maher ER, Guay-Woodford LM, et al: Candidate exome capture identifies mutation of SDCCAG8 as the cause of a retinal-renal ciliopathy. Nat Genet 2010, 42:840-850

9. Walsh $T$, Shahin $H$, Elkan-Miller $T$, Lee MK, Thornton AM, Roeb W, Abu Rayyan A, Loulus S, Avraham KB, King M-C, Kanaan M: Whole exome sequencing and homozygosity mapping identify mutation in the cell polarity protein GPSM2 as the cause of nonsyndromic hearing loss DFNB82. Am J Hum Genet 2010, 87:90-94.

10. Abou Jamra R, Philippe O, Raas-Rothschild A, Eck SH, Graf E, Buchert R, Borck G, Ekici A, Brockschmidt FF, Nöthen MM, Munnich A, Strom TM, Reis A, Colleaux L: Adaptor Protein Complex 4 Deficiency Causes Severe Autosomal-Recessive Intellectual Disability, Progressive Spastic Paraplegia, Shy Character, and Short Stature. The American Journal of Human Genetics 2011, 88:788-795.

11. Sirmaci A, Walsh T, Akay H, Spiliopoulos M, Sakalar YB, HasanefendioğluBayrak A, Duman D, Farooq A, King M-C, Tekin M: MASP1 mutations in patients with facial, umbilical, coccygeal, and auditory findings of Carnevale, Malpuech, OSA, and Michels syndromes. Am J Hum Genet 2010, 87:679-686.

12. Bowden DW, An SS, Palmer ND, Brown WM, Norris JM, Haffner SM, Hawkins GA, Guo X, Rotter Jl, Chen YDI, Wagenknecht LE, Langefeld CD: Molecular basis of a linkage peak: exome sequencing and family-based analysis identify a rare genetic variant in the ADIPOQ gene in the IRAS Family Study. Hum Mol Genet 2010, 19:4112-4120.

13. Musunuru K, Pirruccello JP, Do R, Peloso GM, Guiducci C, Sougnez C, Garimella KV, Fisher S, Abreu J, Barry AJ, Fennell T, Banks E, Ambrogio L, Cibulskis K, Kernytsky A, Gonzalez E, Rudzicz N, Engert JC, DePristo MA, Daly MJ, Cohen JC, Hobbs HH, Altshuler D, Schonfeld G, Gabriel SB, Yue P, Kathiresan S: Exome sequencing, ANGPTL3 mutations, and familial combined hypolipidemia. N Engl J Med 2010, 363:2220-2227.

14. Rosenthal EA, Ronald J, Rothstein J, Rajagopalan R, Ranchalis J, Wolfbauer G Albers JJ, Brunzell JD, Motulsky AG, Rieder MJ, Nickerson DA, Wijsman EM, Jarvik GP: Linkage and association of phospholipid transfer protein activity to LASS4. Journal of Lipid Research 2011, 52:1837-1846.

15. Sobreira NLM, Cirulli ET, Avramopoulos D, Wohler E, Oswald GL, Stevens EL, Ge D, Shianna KV, Smith JP, Maia JM, Gumbs CE, Pevsner J, Thomas G, Valle D, Hoover-Fong JE, Goldstein DB: Whole-Genome Sequencing of a Single Proband Together with Linkage Analysis Identifies a Mendelian Disease Gene. PLoS Genet 2010, 6:e1000991.

16. Anastasio N, Ben-Omran T, Teebi A, Ha KCH, Lalonde E, Ali R, Almureikhi M, Der Kaloustian VM, Liu J, Rosenblatt DS, Majewski J, Jerome-Majewska LA: Mutations in SCARF2 are responsible for Van Den Ende-Gupta syndrome. Am J Hum Genet 2010, 87:553-559.

17. Choi M, Scholl UI, Ji W, Liu T, Tikhonova IR, Zumbo P, Nayir A, Bakkaloglu A Ozen S, Sanjad S, Nelson-Williams C, Farhi A, Mane S, Lifton RP: Genetic diagnosis by whole exome capture and massively parallel DNA sequencing. Proc Natl Acad Sci USA 2009, 106:19096-19101.

18. Götz A, Tyynismaa H, Euro L, Ellonen P, Hyötyläinen T, Ojala T, Hämäläinen RH, Tommiska J, Raivio T, Oresic M, Karikoski R, Tammela O, Simola KOJ, Paetau A, Tyni T, Suomalainen A: Exome Sequencing Identifies Mitochondrial Alanyl-tRNA Synthetase Mutations in Infantile Mitochondrial Cardiomyopathy. American journal of human genetics 2011, 88:635-642.

19. Becker J, Semler O, Gilissen C, Li Y, Bolz HJ, Giunta C, Bergmann C, Rohrbach M, Koerber F, Zimmermann K, de Vries P, Wirth B, Schoenau E, Wollnik B, Veltman JA, Hoischen A, Netzer C: Exome Sequencing Identifies Truncating Mutations in Human SERPINF1 in Autosomal-Recessive Osteogenesis Imperfecta. American journal of human genetics 2011, 88:362-371.

20. Pippucci T, Benelli M, Magi A, Martelli PL, Magini P, Torricelli F, Casadio R, Seri M, Romeo G: EX-HOM (EXome HOMozygosity): A Proof of Principle. Human heredity 2011, 72:45-53.

21. Krawitz PM, Schweiger MR, Rödelsperger C, Marcelis C, Kölsch U, Meisel C, Stephani F, Kinoshita T, Murakami Y, Bauer S, Isau M, Fischer A, Dahl A, Kerick M, Hecht J, Köhler S, Jäger M, Grunhagen J, de Condor BJ, Doelken S, Brunner HG, Meinecke P, Passarge E, Thompson MD, Cole DE, Horn D, Roscioli T, Mundlos S, Robinson PN: Identity-by-descent filtering of exome sequence data identifies PIGV mutations in hyperphosphatasia mental retardation syndrome. Nat Genet 2010, 42:827-829.

22. Rödelsperger C, Krawitz P, Bauer S, Hecht J, Bigham AW, Bamshad M, de Condor BJ, Schweiger MR, Robinson PN: Identity-by-descent filtering of exome sequence data for disease-gene identification in autosomal recessive disorders. Bioinformatics 2011, 27:829-836.

23. Ng SB, Buckingham KJ, Lee C, Bigham AW, Tabor HK, Dent KM, Huff CD, Shannon PT, Jabs EW, Nickerson DA, Shendure J, Bamshad MJ: Exome sequencing identifies the cause of a mendelian disorder. Nat Genet 2010, 42:30-35.

24. Haack TB, Danhauser K, Haberberger B, Hoser J, Strecker V, Boehm D, Uziel G, Lamantea E, Invernizzi F, Poulton J, Rolinski B, luso A, Biskup S, Schmidt T, Mewes H-W, Wittig I, Meitinger T, Zeviani M, Prokisch H: Exome sequencing identifies ACAD9 mutations as a cause of complex I deficiency. Nat Genet 2010, 42:1131-1134.

25. Ng SB, Bigham AW, Buckingham KJ, Hannibal MC, McMillin MJ, Gildersleeve HI, Beck AE, Tabor HK, Cooper GM, Mefford HC, Lee C, Turner EH, Smith JD, Rieder MJ, Yoshiura K-I, Matsumoto N, Ohta T, Niikawa N, Nickerson DA, Bamshad MJ, Shendure J: Exome sequencing identifies MLL2 mutations as a cause of Kabuki syndrome. Nat Genet 2010, 42:790-793.

26. Pierce SB, Walsh T, Chisholm KM, Lee MK, Thornton AM, Fiumara A, Opitz JM, Levy-Lahad E, Klevit RE, King M-C: Mutations in the DBPdeficiency protein HSD17B4 cause ovarian dysgenesis, hearing loss, and ataxia of Perrault Syndrome. Am J Hum Genet 2010, 87:282-288. 
27. Norton N, Li D, Rieder MJ, Siegfried JD, Rampersaud E, Züchner S, Mangos S, Gonzalez-Quintana J, Wang L, McGee S, Reiser J, Martin E, Nickerson DA, Hershberger RE: Genome-wide Studies of Copy Number Variation and Exome Sequencing Identify Rare Variants in BAG3 as a Cause of Dilated Cardiomyopathy. American journal of human genetics 2011, 88:273-282.

28. Glazov EA, Zankl A, Donskoi M, Kenna TJ, Thomas GP, Clark GR, Duncan EL, Brown MA: Whole-Exome Re-Sequencing in a Family Quartet Identifies POP1 Mutations As the Cause of a Novel Skeletal Dysplasia. PLoS Genet 2011, 7:e1002027.

29. Shi Y, Li Y, Zhang D, Zhang H, Li Y, Lu F, Liu X, He F, Gong B, Cai L, Li R, Liao S, Ma S, Lin H, Cheng J, Zheng H, Shan Y, Chen B, Hu J, Jin X, Zhao P, Chen $Y$, Zhang $Y$, Lin $Y$, Li X, Fan Y, Yang H, Wang J, Yang Z: Exome Sequencing Identifies ZNF644 Mutations in High Myopia. PLoS Genet 2011, 7:e1002084.

30. Le Goff C, Mahaut C, Wang LW, Allali S, Abhyankar A, Jensen S, Zylberberg L, Collod-Beroud G, Bonnet D, Alanay Y, Brady AF, Cordier M-P, Devriendt K, Genevieve D, Kiper PÖS, Kitoh H, Krakow D, Lynch SA, Le Merrer M, Mégarbane A, Mortier G, Odent S, Polak M, Rohrbach M, Sillence D, Stolte-Dijkstra I, Superti-Furga A, Rimoin DL, Topouchian V, Unger S, et al: Mutations in the TGF[beta] Binding-Protein-Like Domain 5 of FBN1 Are Responsible for Acromicric and Geleophysic Dysplasias. The American Journal of Human Genetics 2011, 89:7-14.

31. Züchner S, Dallman J, Wen R, Beecham G, Naj A, Farooq A, Kohli MA, Whitehead PL, Hulme W, Konidari I, Edwards YJK, Cai G, Peter I, Seo D, Buxbaum JD, Haines JL, Blanton S, Young J, Alfonso E, Vance JM, Lam BL, Peričak-Vance MA: Whole-Exome Sequencing Links a Variant in DHDDS to Retinitis Pigmentosa. American journal of human genetics 2011, 88:201-206.

32. Kruglyak L, Daly MJ, Reeve-Daly MP, Lander ES: Parametric and nonparametric linkage analysis: a unified multipoint approach. American Journal of Human Genetics 1996, 58:1347-1363.

33. Lander ES, Botstein D: Homozygosity Mapping: A Way to Map Human Recessive Traits with the DNA of Inbred Children. Science 1987, 236:1567-1570.

34. The International HapMap Consortium: A second generation human haplotype map of over 3.1 million SNPs. Nature 2007, 449:851-861.

35. Bahlo M, Bromhead CJ: Generating linkage mapping files from Affymetrix SNP chip data. Bioinformatics 2009, 25:1961-1962.

36. Harismendy O, Ng PC, Strausberg RL, Wang X, Stockwell TB, Beeson KY, Schork NJ, Murray SS, Topol EJ, Levy S, Frazer KA, Harismendy O, Ng PC, Strausberg RL, Wang X, Stockwell TB, Beeson KY, Schork NJ, Murray SS, Topol EJ, Levy S, Frazer KA: Evaluation of next generation sequencing platforms for population targeted sequencing studies. Genome Biology 2009, 10:R32.

37. Cherny SS, Abecasis GR, Cookson WO, Sham PC, Cardon LR: The effect of genotype and pedigree error on linkage analysis: analysis of three asthma genome scans. Genet Epidemiol 2001, 21(Suppl 1):S117-122.

38. Li H, Ruan J, Durbin R: Mapping short DNA sequencing reads and calling variants using mapping quality scores. Genome Research 2008, 18:1851-1858

39. McKenna A, Hanna M, Banks E, Sivachenko A, Cibulskis K, Kernytsky A, Garimella K, Altshuler D, Gabriel S, Daly M, DePristo MA: The Genome Analysis Toolkit: a MapReduce framework for analyzing next-generation DNA sequencing data. Genome Res 2010, 20:1297-1303.

40. Abecasis GR, Wigginton JE: Handling Marker-Marker Linkage Disequilibrium: Pedigree Analysis with Clustered Markers. American journal of human genetics 2005, 77:754-767.

41. Browning SR, Browning BL: High-Resolution Detection of Identity by Descent in Unrelated Individuals. American journal of human genetics 2010, 86:526-539.

42. Thompson EA: Inferring coancestry of genome segments in populations. Invited Proceedings of the 57th Session of the International Statistical Institute; Durban, South Africa 2009.

43. Novoalign. [http://www.novocraft.com].

44. Picard. [http://picard.sourceforge.net].

45. Li H, Handsaker B, Wysoker A, Fennell T, Ruan J, Homer N, Marth G, Abecasis G, Durbin R, Genome Project Data Processing S, Li H, Handsaker B, Wysoker A, Fennell T, Ruan J, Homer N, Marth G, Abecasis G, Durbin R: The Sequence Alignment/Map format and SAMtools. Bioinformatics 2009, 25:2078-2079.
46. Linkdatagen MPS. [http://bioinf.wehi.edu.au/software/linkdatagen/\#mps]

47. Abecasis GR, Cherny SS, Cookson WO, Cardon LR: Merlin-rapid analysis of dense genetic maps using sparse gene flow trees.[see comment]. Nature Genetics 2002, 30:97-101.

48. Wang K, Li M, Hakonarson H: ANNOVAR: functional annotation of genetic variants from high-throughput sequencing data. Nucleic Acids Research 2010, 38:e164

doi:10.1186/gb-2011-12-9-r85

Cite this article as: Smith et al:: Reducing the exome search space for Mendelian diseases using genetic linkage analysis of exome genotypes. Genome Biology 2011 12:R85.

\section{Submit your next manuscript to BioMed Central and take full advantage of:}

- Convenient online submission

- Thorough peer review

- No space constraints or color figure charges

- Immediate publication on acceptance

- Inclusion in PubMed, CAS, Scopus and Google Scholar

- Research which is freely available for redistribution 